\title{
The fractured edentulous atrophic mandible - open or closed treatment?
}

\author{
What is the best way to manage fractured edentulous atrophic mandibles?
}

\author{
Nasser M, Fedorowicz Z, Ebadifar A. \\ Management of the fractured edentulous atrophic mandible. \\ Cochrane Database Syst Rev 2007, issue 1
}

Data sources The Cochrane Oral Health Group Trials Register, Cochrane Central Register of Controlled Trials, Medline and Embase were used for searches. The Internet was searched for potentially relevant meta-analyses and non-Cochrane systematic reviews, ongoing clinical trials and published dissertations. Reference lists of identified studies were cross-checked for any potentially relevant clinical trials. There were no language restrictions.

Study selection Studies were selected if they were randomised controlled trials involving people over 55 years of age with fractures in the symphysis, parasymphysis, body, angle, ramus, condyle, and coronoid process of atrophic edentulous mandibles in which the fracture was a result of trauma, implant insertion or due to pathological fracture. Any studies that compared methods of management (open or closed reduction or fixation) were selected.

Data extraction and synthesis Screening of eligible studies was conducted in duplicate and independently by two review authors. It was intended to express results as random-effects models using mean differences for continuous outcomes and risk ratios for dichotomous outcomes with $95 \%$ confidence intervals. Heterogeneity was to be investigated including both clinical and methodological factors.

Results No eligible randomised controlled trials were identified.

Conclusions This review illustrates that there is currently inadequate evidence to support the effectiveness of any single approach, either open or closed, in the management of fractured atrophic edentulous mandibles and that, until high-level evidence is available, treatment decisions should continue to be based on clinician's prior experience. This absence of evidence may in part reflect a certain lack of clarity and the apparent diversity and lack of reliability in some of the traditional and normative predictors of successful outcomes.

\section{Commentary}

The authors have attempted to address an important issue over which there is much debate. It is disappointing but not surprising that there are no randomised controlled studies investigating the effectiveness of open or closed interventions, and that therefore the authors were not able to begin to meet their objectives. The review would have been improved if it had included noninterventional treatment options in the management of this group of patients. The literature comparing this approach with interventional treatments is of similar quality to the literature reviewed by the authors.

From a general practice point of view, the treatment options can result in a number of issues that may influence provision of a lower denture. These are essentially irregular contour at the fracture sites, if treated conservatively or with closed methods. If fractures are opened and plated this can result in the plate position interfering with subsequent lower dentures.

The criterion for inclusion of studies to be considered is very reasonable and the search methodology to identify relevant studies is thorough. The assessment of papers identified for possible inclusion in the review and the assessment of the quality of those papers is robust. The reasons for exclusion of papers are well-documented. The information available from papers that were studied is well-summarised.

The discussion is a fair assessment of the current situation relating to the active treatment of edentulous fractures: given the title of the review, however, if considering the management of the edentulous mandible, it is weakened by the absence of any information on a non interventional management approach, ie, simply treating patients with soft diet and analgesia. The latter is a very viable option for a large number of edentulous fractures because many of them are not compound. Although this approach may not achieve a good reduction, because the patients are edentulous, reduction to restore occlusion is not necessary since the occlusion can be corrected with new dentures. A large number of people can function just as well if healing occurs with a poor reduction. This non interventional approach removes all operative risk from a group of patients who can often be at risk of complications from any sort of operative intervention that requires general anaesthesia.

The authors provide a useful list of references on the subject and information on details used in the review and forming its conclusions.

\section{Ian Holland \\ Maxillofacial Department, Southern General Hospital, Glasgow, Scotland, UK}

Evidence-Based Dentistry (2007) 8, 5-6. doi:10.1038/sj.ebd.6400515
Address for correspondence: Luisa Fernandez, Group Co-ordinator, Cochrane Oral Health Group, MANDEC, 3rd Floor Dental Hospital, University of

Manchester, Higher Cambridge Street, Manchester M15 6FH, UK.

E-mail luisa.fernandez@manchester.ac.uk 